\title{
Characteristics of Tetanic Force Produced by the Sternomastoid Muscle of the Rat
}

\author{
Stanislaw Sobotka ${ }^{1,2}$ and Liancai $\mathrm{Mu}^{1}$ \\ ${ }^{1}$ Department of Research, Upper Airway Research Laboratory, Hackensack University Medical Center, Hackensack, NJ 07601, USA \\ ${ }^{2}$ Department of Neurosurgery, Mount Sinai School of Medicine, NY 10029, USA
}

Correspondence should be addressed to Stanislaw Sobotka, ssobotka@humed.com

Received 2 November 2009; Revised 9 February 2010; Accepted 4 March 2010

Academic Editor: Henk L. M. Granzier

Copyright ( $) 2010$ S. Sobotka and L. Mu. This is an open access article distributed under the Creative Commons Attribution License, which permits unrestricted use, distribution, and reproduction in any medium, provided the original work is properly cited.

The sternomastoid (SM) muscle plays an important role in supporting breathing. It also has unique anatomical advantages that allow its wide use in head and neck tissue reconstruction and muscle reinnervation. However, little is known about its contractile properties. The experiments were run on rats and designed to determine in vivo the relationship between muscle force (active muscle contraction to electrical stimulation) with passive tension (passive force changing muscle length) and two parameters (intensity and frequency) of electrical stimulation. The threshold current for initiating noticeable muscle contraction was $0.03 \mathrm{~mA}$. Maximal muscle force $(0.94 \mathrm{~N})$ was produced by using moderate muscle length/tension $(28 \mathrm{~mm} / 0.08 \mathrm{~N}), 0.2 \mathrm{~mA}$ stimulation current, and $150 \mathrm{~Hz}$ stimulation frequency. These data are important not only to better understand the contractile properties of the rat SM muscle, but also to provide normative values which are critical to reliably assess the extent of functional recovery following muscle reinnervation.

\section{Introduction}

The sternocleidomastoid (SCM) muscle lies on the lateral side of the neck. Anatomically, it is composed of two bellies, a medially and superficially localized sternomastoid (SM), and a laterally and deeply positioned cleidomastoid (CM). Functionally, the SCM participates in head movements and respiration [1]. In respiration, it serves as an "accessory" inspiratory muscle in the neck. Activation of the SCM causes cranial displacement of the sternum and ribcage during conscious inspiratory efforts [2-4]. In general, the SCM is not active during resting breathing, but contracts during strong respiratory efforts [5]. Previous studies demonstrated that the SCM plays a particularly important role in patients with obstructive lung disease, where its increased activity even at rest improves oxygen delivery to the lungs [2].

As the SM belly is located more superficially in the neck and has a relatively larger muscle mass when compared with the CM belly, it has been widely used as a muscle or myocutaneous flap for reconstruction of oral cavity and facial defects $[6,7]$. In addition, the SM muscle [8] and cervical strap muscles $[9,10]$ have been commonly used in laryngeal and facial reinnervation.

We have a longstanding interest in the development of novel surgical techniques to effectively reinnervate paralyzed muscles as the presently used reinnervation methods result in poor outcomes (for review see $[11,12]$ ). Although the nerve-muscle pedicle (NMP) technique has been commonly employed to treat laryngeal and facial paralysis in animal experiments and clinical practice, controversy exists concerning the optimal results and success rate of the functional recovery [13-16]. In our on-going reinnervation studies, the SM muscle has been chosen as a studied muscle in a rat model because this muscle has anatomical advantages over other neck muscles. Specifically, the SM muscle and its innervating nerve can be easily accessed and manipulated. In addition, we have established a large database regarding the patterns of nerve supply, motor endplate morphology, and muscle fiber-type distribution of the SM muscle in the rat (unpublished data) which is critical for designing new reinnervation procedures. 
A number of morphological and physiological approaches have been used to assess the success of axonal regeneration and the extent of functional recovery of a reinnervated muscle after a given reinnervation procedure. Electromyography (EMG) $[17,18]$ and muscle force measurement [19-22] are often used to assess functional recovery after muscle reinnervation. The amplitude and frequency of the recorded EMG bursts are indicative of the quantity of the activating motor units involved in a given motor task. However, the maximum force provides a better overall estimate of the mechanics of a whole muscle, and the muscle force measurements are usually used to evaluate quantitatively the mechanical function and contractile properties of a reinnervated muscle.

Although some researchers investigated in vivo SM muscle force in rabbits [23], the force characteristics of the SM muscle in rats have never been determined. Measuring the force of isometric tetanic muscle contraction can be an invaluable tool to evaluate muscle strength after nerve injury and subsequent repair $[21,24]$. Intraspecimen comparison seems to be a practical method for evaluating the recovery of maximum force. It has been recognized that opposite muscles have the same strength in healthy animals [25]. However, after unilateral injury, the left-right muscle balance is not present any more. The healthy muscle is overstrained, as it is now responsible for the constant support of functions previously maintained by muscles from both sides. It leads to changes in the anatomical and physiological characteristics of the neuromuscular system at the noninjured side. Therefore, interspecimen normative data are needed for a nonbiased evaluation of the degree of recovery in a reinnervated muscle.

The present study is focused on determining the muscle force characteristics of the SM muscle in healthy rats. These results would provide normative data which could be useful for understanding the physiological role of the SM muscle and for evaluating the extent of functional recovery after reinnervation of a paralyzed SM muscle. We would also like to establish the optimal stimulation parameters which could be used to produce the strongest isometric force by this muscle.

\section{Materials and Methods}

2.1. Animals. Twelve adult (3.5 months old) Sprague-Dawley male rats (Charles River Laboratories, MA), weighing 350450 grams, were used in this study. Previous studies [26] showed that there is no gender difference in rat upper airway muscle force and other muscle contractile properties. The animals were provided with ad libitum access to food and water and housed in standard cages in a $22^{\circ} \mathrm{C}$ environment with a 12:12-h light-dark cycle. All experimental procedures were reviewed and approved by the Institutional Animal Care and Use Committee prior to the onset of experiments. The experiments were performed in accordance with the Guide for Care and Use of Laboratory Animals published by the US National Institutes of Health (NIH Publication no. 85-23, revised 1996). All efforts were made to minimize the number of animals and their suffering in the experiments.
2.2. Surgical Procedures. All rats underwent open neck surgery under an Olympus SZX12 Stereo zoom surgical microscope (Olympus America Inc., Center Valley, PA) to expose the right SM muscle through a midline skin incision from the hyoid bone to the sternum. Animals were anesthetized with an initial intraperitoneal injection of ketamine ( $80 \mathrm{mg} / \mathrm{kg}$ body wt) and xylazine ( $5 \mathrm{mg} / \mathrm{kg}$ body wt); supplementary doses were administered as needed to maintain an adequate state of anesthesia. The rat was placed on a heating pad (homeothermic blanket system, Stoelting, Wood Dale, IL) to maintain its temperature at $35^{\circ} \mathrm{C}$.

2.3. Muscle Preparation. Our studies have demonstrated that the rat SM is supplied by a branch derived from the spinal accessory nerve and has a single motor endplate band at the midpoint of the muscle (data not shown). The right SM muscle and its innervating nerve were isolated from surrounding tissues and prepared for nerve stimulation and force measurement. First of all, the rostral tendon of the SM was identified, transected, and attached with a 20 suture to a servomotor lever arm (Model 305B DualMode Lever Arm System, Aurora Scientific Inc., Aurora, Ontario, Canada, see Figure 1). The adjustable arm of the servomotor was used to alter muscle length and to provide a measure of muscle force. Then, the SM nerve was placed on a bipolar stimulating electrode constructed from two hooked silver wires separated by $4 \mathrm{~mm}$ (Figure 1) attached to a high precision micromanipulator (Narishige Scientific Instruments, Tokyo, Japan).

2.4. Nerve Stimulation and Muscle Force Measurement. Isometric contraction of the SM muscle was obtained with two $200 \mathrm{~ms}$ trains of biphasic rectangular pulses $(0.2 \mathrm{msec}$ duration) separated by a 20 -second break. A break of at least 1 minute was used before trying subsequent pairs of trains. The maximum value of muscle force during each $200 \mathrm{~ms}$ contraction was identified. The maximal force during the first and second stimulation trains was averaged. Initial passive tension before stimulation was subtracted from this value. This difference between output force and preload force represents the muscle force measurement. Typically, current was set to $0.1 \mathrm{~mA}$ and frequency of stimulation to $200 \mathrm{~Hz}$. The force generated by the contraction of the SM muscle was transduced with the servomotor of a 305B lever system and displayed on a computer screen. At the moment of force measurements, the lever arm was stationary.

To prevent cooling and drying, the SM muscle and nerve were regularly bathed with warm mineral oil throughout the testing. Although changes in muscle temperatures above $25^{\circ} \mathrm{C}$ significantly influence twitch force, they only have a small influence on tetanic force $[27,28]$. To reduce the variability of collected force data, the temperature of the SM muscle was monitored regularly and maintained between 35-36 ${ }^{\circ}$.

During force measurement, several parameters influencing force production were examined to establish the optimal settings for obtaining maximum muscle force as described below. 


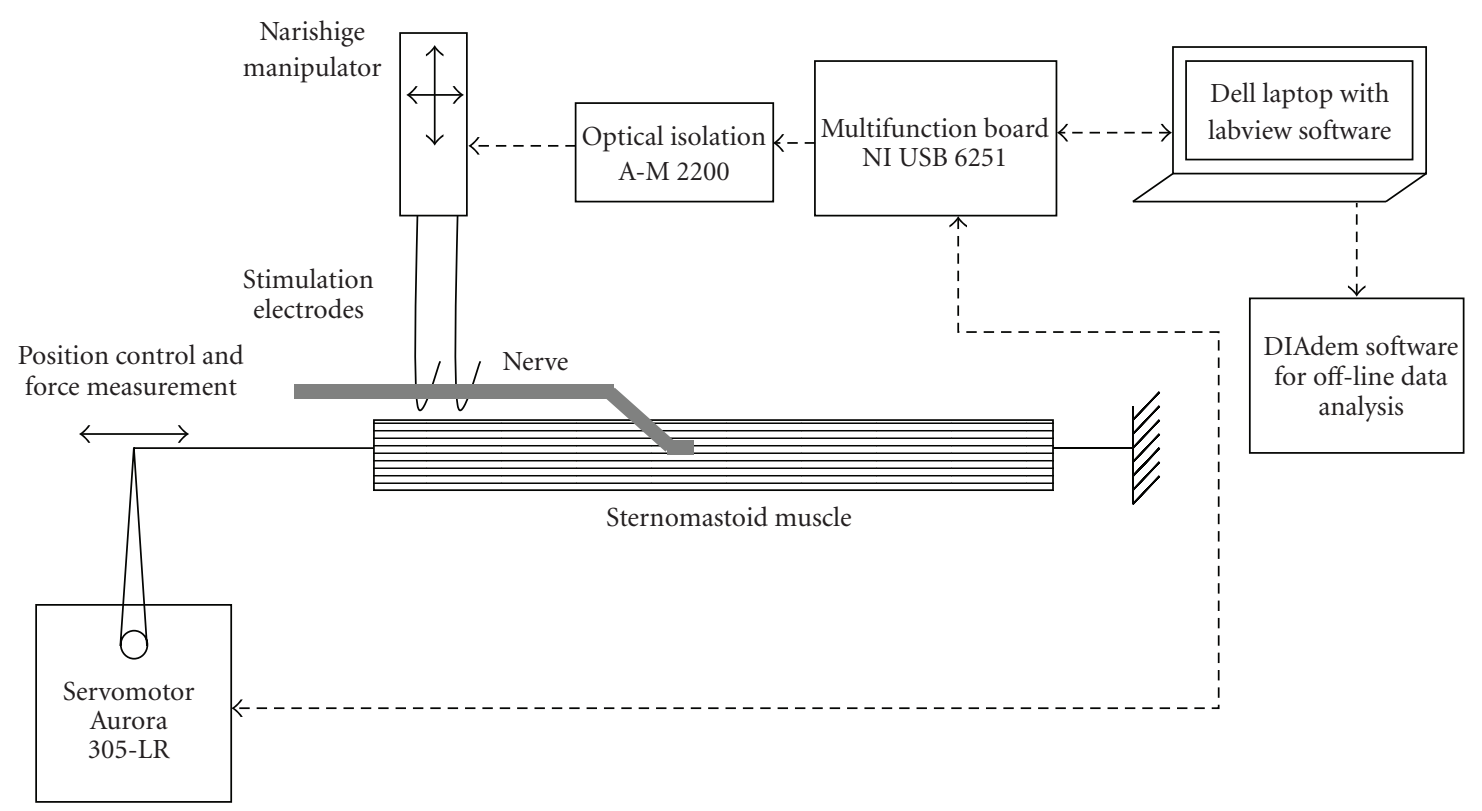

Figure 1: A diagram of the data acquisition system, which provides electrical stimulation and records muscle force. Note that a dell laptop with user written software in Labview 8.2 is used to control the experiment. The SM muscle is detached from its rostral tendon and attached to the lever of servomotor, which controls muscle stretch and measures muscle force. Electrical stimulation with parameters controlled by LabView software (National Instruments) is generated by the Multifunctional board 6251 (National Instruments) and delivered to the SM nerve. Data are analyzed off line with DIAdem 11.0 software (National Instruments).

2.4.1. Muscle Length/Tension. As maximal muscle force can be generated at optimal muscle length, we examined the length-force relationship. Muscle length was controlled by gradually stretching the SM muscle using the lever arm to pull the muscle with different tensions (very low tension$0.04 \mathrm{~N}$, low tension- $-0.06 \mathrm{~N}$, medium tension- $0.08 \mathrm{~N}$, high tension-0.1 N, and very high tension-0.24N). Finally, the length of the muscle (in millimeters) was measured at different levels of passive tension $(0.04-0.24 \mathrm{~N})$. These passive tension values were chosen based on our preliminary studies. The optimal tension that generates the highest muscle force was determined by our preliminary work and confirmed in the experimental group presented in this paper. The muscle force at a given muscle length was measured in response to electrical stimulation with a train of pulses of $0.1 \mathrm{~mA}$ current, at a frequency of 200 pulses per second.

2.4.2. Stimulation Intensity. The SM muscle was stretched with the medium tension of $0.08 \mathrm{~N}$ at which the highest muscle force was consistently produced. Then, the muscle force was measured as a function of stimulation current. The intensity of stimulation current was increased starting from $0.01 \mathrm{~mA}$ through the level where the muscle force reached a plateau (about $0.1 \mathrm{~mA}$ ) and continued at the supramaximal level until $0.5 \mathrm{~mA}$.

2.4.3. Stimulation Frequency. To analyze the other parameters responsible for maximum force generation, a forcefrequency curve was built. Two trains of stimuli $(200 \mathrm{msec}$ duration each, with a rest period of 20 seconds between contractions) with incrementally increasing frequencies were delivered. The stimulation frequency was increased gradually from $5 \mathrm{~Hz}$ (when only one pulse during the $200 \mathrm{~ms}$ stimulation period was given and could be used to evaluate twitch muscle force), through frequencies for which the muscle force reached a plateau (about $100 \mathrm{~Hz}$ ) and continued to increase until $500 \mathrm{~Hz}$.

2.5. Muscle Weight. Following the completion of isometric force testing, the rat was euthanized with an overdose of anesthetic. The entire SM muscle was removed and weighed (in grams).

2.6. Data Acquisition System. The experiment was controlled by an Acquisition System built from a multifunction I/O National Instruments Acquisition Board (NI USB 6251, 16 bit $1.25 \mathrm{Ms} / \mathrm{s}$, National Instruments, Austin, TX) connected to a DELL laptop with a custom written program using labVIEW 8.2 software (National Instruments, see Figure 1). The system produced two output signals with all parameters set by the user through virtual control knobs created by the LabView program. One output provided stimulation pulses, which after isolation from the ground through an optical isolation unit (Analog Stimulus Isolator Model 2200, AM Systems, Inc, Carlsborg, WA) were used for the current controlled nerve stimulation. The other output provided a position signal, which was used by the servomotor of the 305B Dual-Mode Lever System to control muscle length. The Acquisition System was also used to collect a muscle force signal from the 305B Dual-Mode Lever System. Collected data were analyzed offline with DIAdem 11.0 software (National Instruments). 
2.7. Statistical Analysis. Experimental variables included three independent variables (initial passive tension before stimulation, current and frequency of stimulation) and one dependent variable: muscle force generated during stimulation. Minimal stimulation current (when the stimulation train was set at $200 \mathrm{~Hz}$ ), minimal stimulation frequency (when stimulation pulses were set at $0.1 \mathrm{~mA}$ ), and optimal length of the muscle (when stimulation parameters were set at $0.1 \mathrm{~mA}$ and $200 \mathrm{~Hz}$ ), which were able to produce maximal tetanic muscle contraction, were described by the means and standard deviations. The $t$-test for pairs was used to determine the statistical significance of difference between data points. The significance level was set at $P<.05$.

\section{Results}

SM muscle force was defined as the difference between maximal muscle contraction observed during electrical stimulation (with $200 \mathrm{~ms}$ train of pulses) and the initial tension of the muscle just before stimulation. Our goal was to establish optimal muscle length and characteristics of muscle force generated by electrical stimulation, which could be used in our further studies as a reference (muscle force generated by the muscle with an intact nerve) to evaluate the level of muscle force recovery after reinnervation. Therefore, we evaluated how muscle force depends on initial passive tension and how it changes with intensity and frequency of nerve stimulation.

\subsection{Optimal Muscle Length/Tension for Maximal Muscle} Force. Muscle force is a function of muscle length produced by an initial stretch of the muscle before electrical stimulation. The muscle was stretched with the following tensions before stimulation: very loose $(0.04 \mathrm{~N})$, loose $(0.06 \mathrm{~N})$, moderate $(0.08 \mathrm{~N})$, tense $(0.1 \mathrm{~N})$, and very tense $(0.24 \mathrm{~N})$. We used $0.1 \mathrm{~mA}$ pulses at $200 \mathrm{~Hz}$. The averaged data from the whole group of rats illustrating the decrease of muscle force at different passive tensions is shown in Figure 2. The typical length-force "inverted U" relationship was found as described by others $[29,30]$.

The muscle force was found to be the highest (mean $=0.94 \mathrm{~N}$ ), when the muscle was initially stretched at a moderate tension ( set at $0.08 \mathrm{~N}$ ). Decreasing initial tension to "loose" ( set at $0.06 \mathrm{~N}$ ) decreased muscle force by $12 \%$ $(0.82 \mathrm{~N}$-statistically significant decrease $P<.01, t=3.2$, two-tailed $t$-test for pairs, $d f=11$ ), whereas increasing initial tension to "tense" (set at $0.1 \mathrm{~N}$ ) reduced muscle force by $6 \%(0.88 \mathrm{~N}$-not statistically significant decrease, $P>.05$, $t=0.5, d f=11)$.

\subsection{Muscle Force Evoked by Different Intensity of Stimulation.}

To examine the relationship between muscle force and stimulation current, we varied the current from 0 to $0.5 \mathrm{~mA}$ at $200 \mathrm{~Hz}$ trains of pulses when the muscle was stretched with moderate tension of $0.08 \mathrm{~N}$, which produced optimal muscle length. Figure 3 shows the time course of muscle force responses to different stimulation currents in a representative rat. The difference between the maximal force produced by

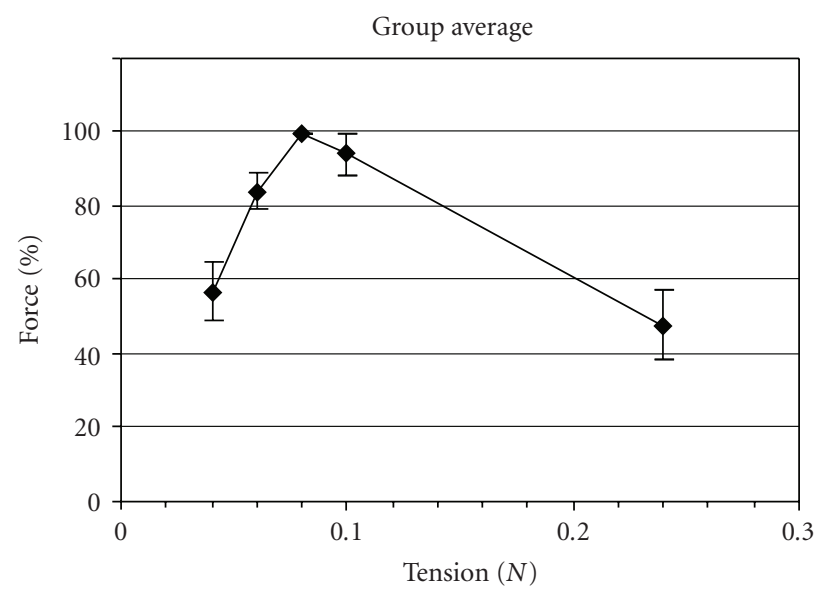

Figure 2: Muscle force as a function of passive tension before stimulation. This force-tension curve was normalized by maximal force to illustrate the rate of decline of force at different passive tensions (set up just before electrical stimulation). The nerve was stimulated with $0.1 \mathrm{~mA}$ pulses at $200 \mathrm{~Hz}$. The group average is shown. Vertical bars represent standard error of the mean. Nerve stimulation at moderate tension of the muscle $(0.08 \mathrm{~N})$ yielded maximal muscle force $(0.94 \mathrm{~N})$. The data presented in this and all following figures were collected, when the nerve was stimulated with a $200 \mathrm{~ms}$ train of biphasic pulses of $0.2 \mathrm{~ms}$ width.

the SM muscle during nerve stimulation and the passive tension before stimulation was used to generate the currentforce curve. The relationship between the density of force produced by the SM muscle (normalized by it's crosssection area) and stimulation current in the group average is shown in Figure 4 . In most of our animals, $0.03 \mathrm{~mA}$ was the threshold current, which produced noticeable muscle contraction. Contraction force gradually increased with an increase of stimulation current until reaching the level of maximal muscle force at a stimulation current between $0.1 \mathrm{~mA}$ and $0.2 \mathrm{~mA}$. In most of our animals, increasing stimulation current from $0.1 \mathrm{~mA}$ to $0.2 \mathrm{~mA}$ still produced an increase in muscle force (in average 11\% increasestatistically significant increase $P<.05, t=2.3$, two-tailed $t$-test for pairs, $d f=11$ ). Further increases of stimulation current did not increase muscle force.

3.3. Muscle Force as a Function of Stimulation Frequency. We also analyzed how muscle force changes with regard to the frequency of stimulation pulses (from 0 to $500 \mathrm{~Hz}$ ). We used a $200 \mathrm{~ms}$ train of $0.1 \mathrm{~mA}$ pulses. The muscle was stretched with a moderate tension $(0.08 \mathrm{~N})$. Figure 5 illustrates in a representative rat the muscle force in response to 6 different frequencies of stimulation (maximal values of force for each frequency were measured to create a frequency-force curve). Stimulation pulses below $25 \mathrm{~Hz}$ produced individual twitches of the muscle in response to each pulse separately, with a small summation of responses observed already at $25 \mathrm{~Hz}$. The frequency-density relationship of muscle force (normalized by cross-section area of the muscle) in the group data is shown in Figure 6. Muscle force increased with stimulation frequency, starting at $25 \mathrm{~Hz}$ (with almost a full tetanic fusion 

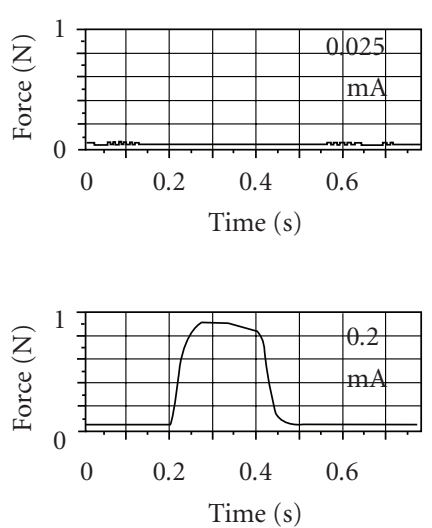

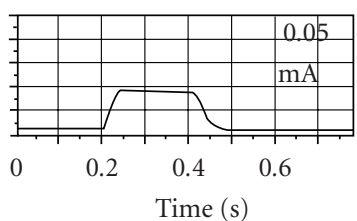

(a)

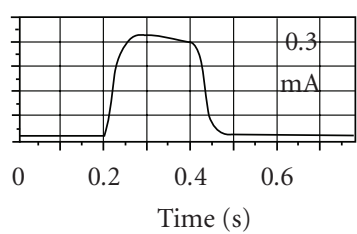

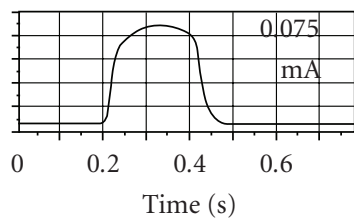

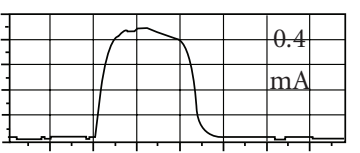

0
0.2 Time (s)
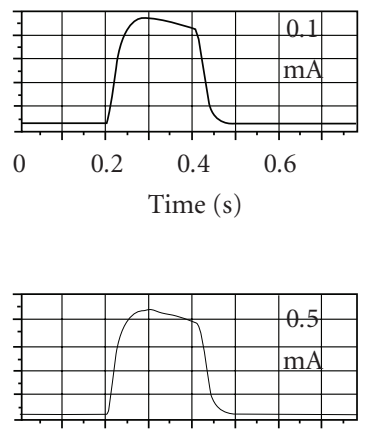

$\begin{array}{llll}0 & 0.2 & 0.4 & 0.6\end{array}$

(b)

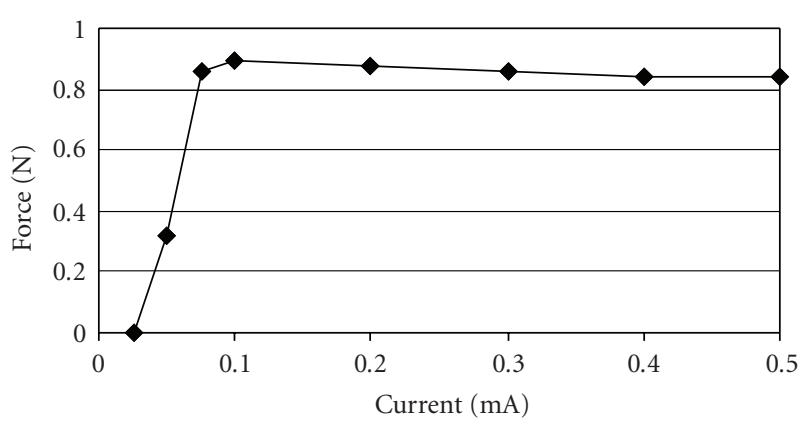

(c)

FIGURE 3: Force measurements from a representative rat, showing the stimulation intensity-force relationship. (a and b) show the time course of muscle force produced by electrical stimulation of the SM nerve at eight different intensities. Note that stimulation at $0.1 \mathrm{~mA}$ resulted in maximal muscle contraction. (c) illustrates the outcome from these 8 measurements - the relationship between muscle force and stimulation intensity.

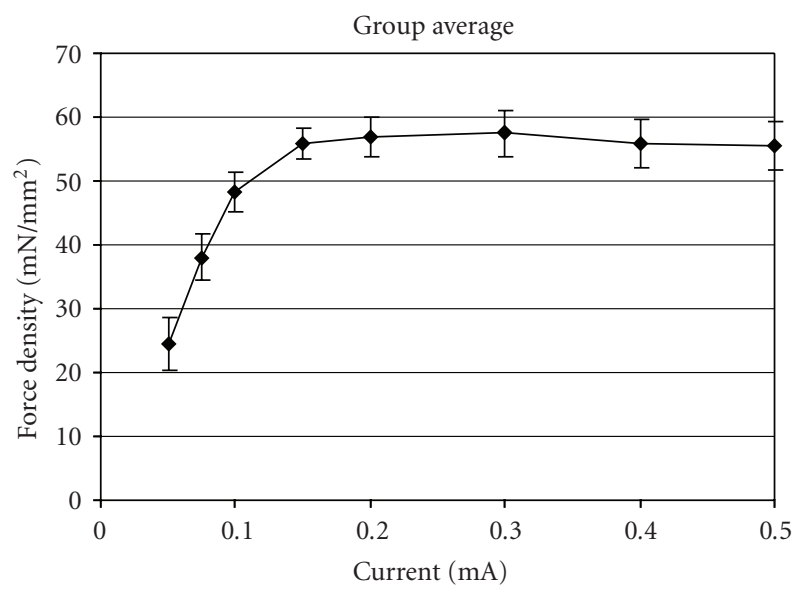

Figure 4: Muscle force as a function of stimulation current. This group average shows the density of force produced by the SM muscle and normalized by its cross-section area at different stimulation currents. Vertical bars represent the standard error of the mean. The passive tension was at a moderate level $(0.08 \mathrm{~N})$. The nerve was stimulated at $200 \mathrm{~Hz}$.

of force at $50 \mathrm{~Hz}$ ) and reached maximal value at $150 \mathrm{~Hz}$. Further increases of stimulation frequency (above $300 \mathrm{~Hz}$ ) produced a small but consistent decrease of muscle force. The muscle force generated by the stimulation train of $500 \mathrm{~Hz}$ (the highest frequency used in this study) was $21 \%$ smaller than that generated by the stimulation of $150 \mathrm{~Hz}$. The difference was statistically significant $(P<.01, t=$ 3.5 , two-tailed $t$-test for pairs, $d f=11$ ). A similar shape of the frequency-force relationship was seen for different stimulation currents (see Figure 7).

3.4. Muscle Length and Weight. Immediately after the experimental session, the length of the SM muscle was measured at different stretching forces, and then the muscle was removed and weighed. The average length was $25.7 \mathrm{~mm}$ (range 24$27 \mathrm{~mm})$ at very loose tension $(0.04 \mathrm{~N}), 26.8 \mathrm{~mm}$ at loose tension $(0.06 \mathrm{~N}), 27.7 \mathrm{~mm}$ at moderate tension $(0.08 \mathrm{~N})$, $28.6 \mathrm{~mm}$ at tense $(0.1 \mathrm{~N})$, and $31.4 \mathrm{~mm}$ at very tense $(0.24 \mathrm{~N})$. The average muscle weight was $0.50 \mathrm{~g}$ (range $0.47-0.53 \mathrm{~g}$ ).

\section{Discussion}

This study investigated the muscle force features of the $\mathrm{SM}$ muscle in a rat model. We determined the correlations of muscle force (active muscle contraction to electrical stimulation) with passive tension (passive force changing muscle length) and two parameters of electrical stimulation, 


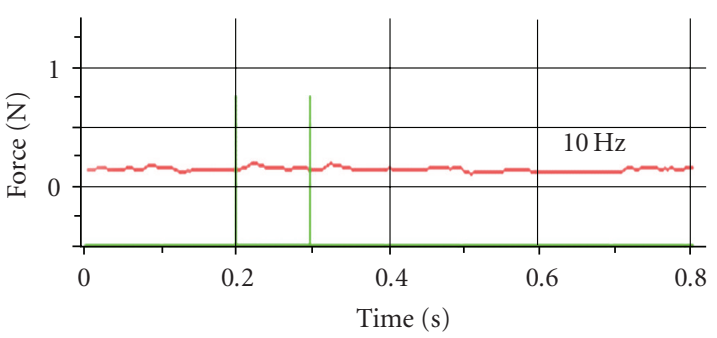

(a)

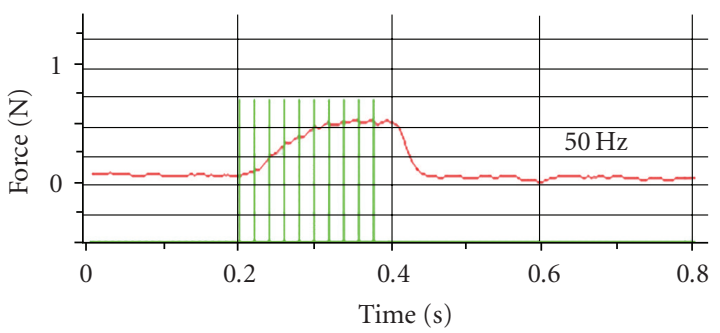

(c)

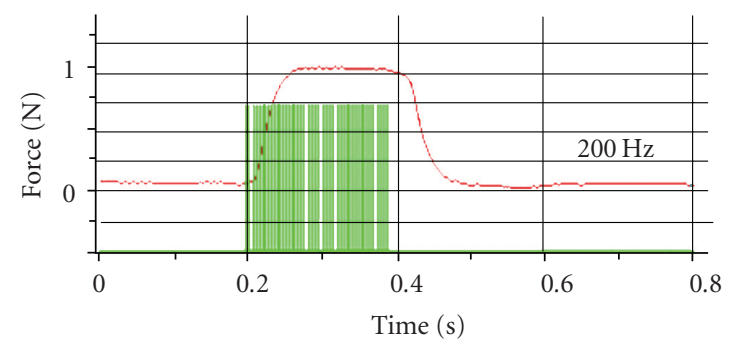

(e)

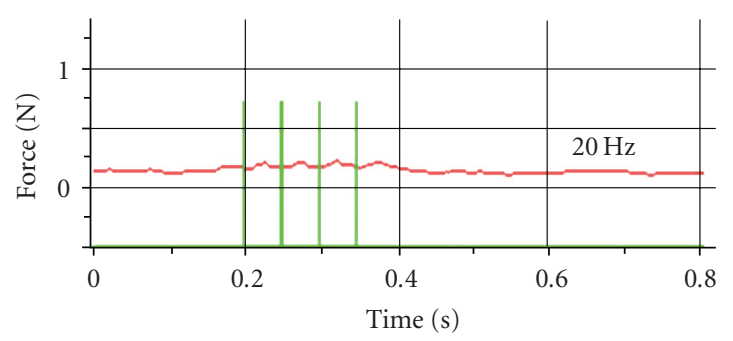

(b)

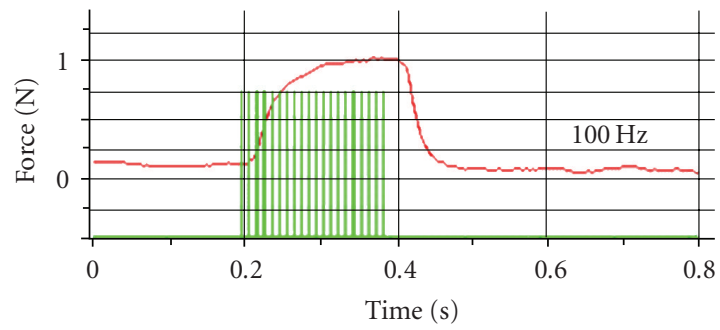

(d)

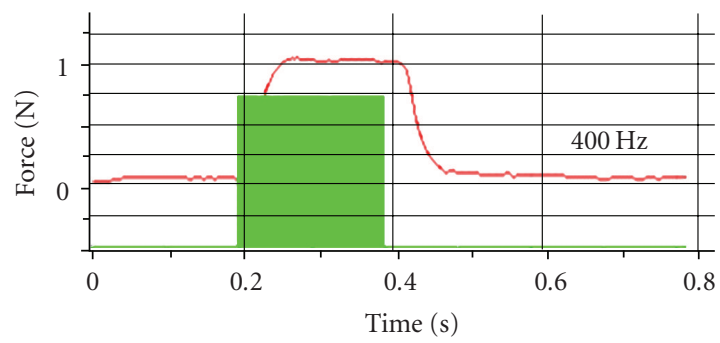

(f)

FIgURE 5: Illustration of muscle force measurement as a function of stimulation frequency in a representative rat. Individual muscle contractions to stimulation pulses at different frequencies are shown in red. Single stimulation pulses are indicated by green vertical lines. The muscle responded with single twitches until $25 \mathrm{~Hz}$. At $50 \mathrm{~Hz}$ the muscle contractions were fused. With an increasing frequency of stimulation, the muscle responded with increased force, which reached a plateau at about $150 \mathrm{~Hz}$.

intensity and frequency. There are several key findings of this study. First of all, moderate muscle length/tension $(28 \mathrm{~mm} / 0.08 \mathrm{~N})$ produced maximal muscle force $(0.94 \mathrm{~N})$. Second, $0.03 \mathrm{~mA}$ was the threshold current for initiating noticeable muscle contraction. Third, $0.2 \mathrm{~mA}$ was the stimulation current which produced the maximal force in the SM. Finally, the stimulation frequency that produced maximal muscle force was about $150 \mathrm{~Hz}$. Taken together, in the normal rat, maximal force in the SM can be produced with moderate passive tension, $0.2 \mathrm{~mA}$ current, and $150 \mathrm{~Hz}$ frequency. These findings are important not only for better understanding the contractile properties of the rat SM muscle, but also for providing normative values which would be useful for reliably evaluating the extent of functional recovery induced by muscle reinnervation.

4.1. Passive Tension-Muscle Force Relationship. Muscle length is an important variable affecting active muscle force generated in response to electrical stimulation. However, establishing the optimal length of the muscle, which could produce maximal muscle force, requires lengthy investigation at many different lengths each time a new muscle is studied.
The present study shows the highest SM muscle force when the muscle is stretched with a tension of $0.08 \mathrm{~N}$ before stimulation ( $8.5 \%$ of maximal isometric tetanic force). Data from this study showed the typical "U shape" relationship between length and force in the SM muscle. Our results are consistent with the general characteristics obtained in muscle force measurements where other muscles were studied in the rat and other species $[23,25]$. Optimal muscle length also varies with stimulation frequency. A higher optimal muscle length was found for lower stimulation frequencies as described [29, 31]. A straightforward and efficient method to stretch a muscle to optimal length, which would result in optimal active muscle force, is to apply a passive force to the muscle with the previously established tension. Therefore, we analyzed the relationship between passive tension stretching a muscle and active force generated by the muscle in response to electrical stimulation.

Previous studies showed a very wide range of optimal passive tensions in different muscles and species which allow muscles to be stretched to optimal length and contract with maximal force. Celichowski et al. [32] stretched the rat's medial gastrocnemius muscle up to a passive tension 


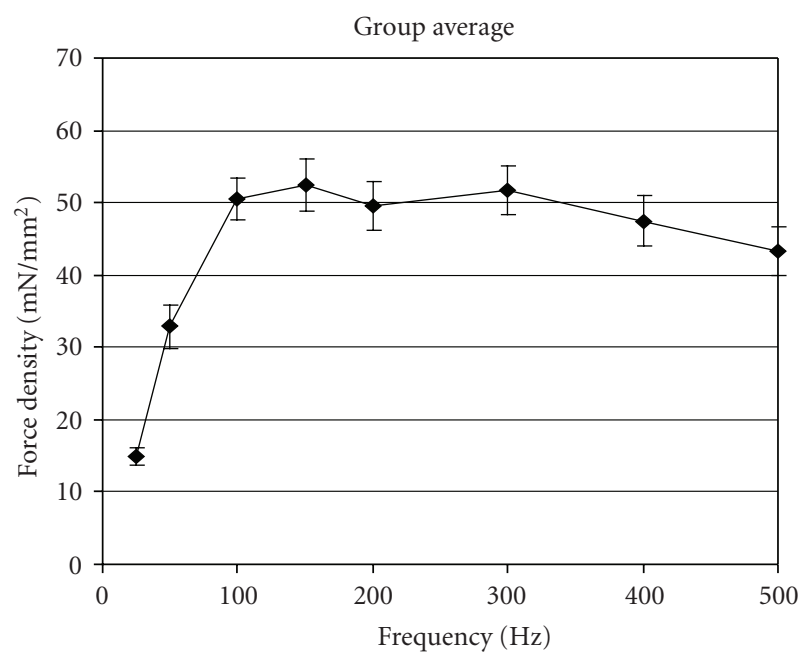

Figure 6: Muscle force as a function of stimulation frequency. The group data shows the relationship between muscle force density (normalized to cross-section area) and stimulation frequency. Vertical bars represent standard error of the mean. The passive tension was at a moderate level $(0.08 \mathrm{~N})$. The nerve stimulation current was $0.1 \mathrm{~mA}$.

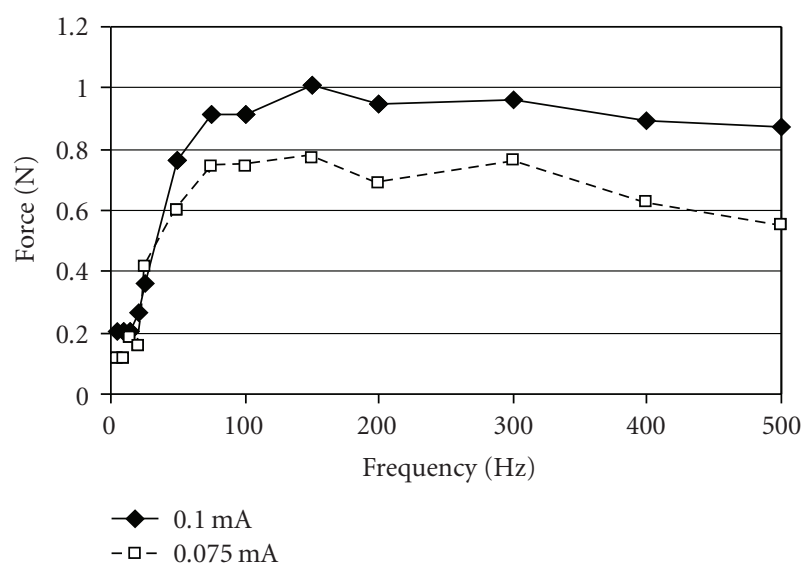

Figure 7: A representative example in an individual rat to show the relation between muscle force and stimulation frequency at two levels of stimulation current. The passive tension was at a moderate level $(0.08 \mathrm{~N})$. The nerve stimulation current was $0.1 \mathrm{~mA}$ (continuous line) or $0.075 \mathrm{~mA}$ (dashed line). Muscle force is higher for a bigger current but otherwise both curves share similar characteristics with a slight decline of force for the highest frequency of stimulation.

of $100 \mathrm{mN}$ to get muscle contraction with maximal force. Johns et al. [33] studied the length-tension relationships in the thyroarytenoid and digastric muscles of the cat. They showed that the thyroarytenoid muscle requires $0.14 \mathrm{~N}$ of passive tension (39\% of maximal isometric tetanic force) to stretch the muscle to optimum length (Lo), whereas the digastric muscle requires a much smaller $0.028 \mathrm{~N}$ of passive tension ( $9 \%$ of maximal isometric tetanic force). The authors claimed that a large passive tension of the thyroarytenoid muscle is needed to allow a modulation of tension in the vocal cord during phonation. The underlining mechanism can be also related to a considerable amount of connective tissue in parallel with the muscle fibers. Krier et al. [34] found substantial passive tension in the striated muscle of the external anal sphincter when the muscle was stretched to optimal muscle length (12\% of active isometric tetanus tension). The authors hypothesized that the substantial passive tension of this muscle provides a sphincteric contractile tone and plays a role in the maintenance of fecal continence. Floyd and Morrison [35] studied cat and sheep esophageal striated muscle strips. They found that the passive tension at the optimal length is equal to $10 \%$ of the active isometric contraction. Kim et al. [36] studied the canine diaphragm. The optimal muscle length (for maximal force) was 125\% of the muscle length at which passive tension was noticed for the first time. At the optimal length, resting tension was $12 \%$ of active muscle force. The authors speculated that their diaphragm's length-tension curve may represent an evolutionary adaptation to the volume and pressure requirements of mammalian respiration. The position of the length-passive tension curve with respect to the length-active tension curve might also depend on the amount of elastic material in the muscle [37]. Therefore, the removal of a substantial amount of connective tissue from a muscle for testing the muscle outside a body may also lead to different length-passive tension curve (as compared to testing the same muscle in vivo). Farkas and Rochester [38] showed that the canine SM and other inspiratory muscles do not share common length-tension properties or resting lengths. The muscles modify different resting lengths with lung volume and body position. These changes in muscle lengths influence muscle force generating capacity.

Large muscle stretching during measurement could have a detrimental impact on subsequent measurements due to stretch-induced damage. Davis et al. [39] reported lengthtension data from the rabbit tibialis anterior. They used excessive tensions and showed that passive muscle force grows with an increase of muscle length until reaching almost $(92 \%)$ of maximal active muscle force $(12 \mathrm{~N})$ and then starts to decrease. The authors speculated that the decrease in passive muscle force is "presumably due to injury of passive muscle structures such as the surrounding connective tissue or intracellular parallel structures". In our study, maximal passive tension was only $0.24 \mathrm{~N}$, about $25 \%$ of maximal active force generated by the SM muscle $(0.94 \mathrm{~N})$, which is considerably less than $92 \%$ of the passive tension limit beyond which Davis et al. observed decline in passive force. A tension of $0.24 \mathrm{~N}$ when recalculated per the relatively large muscle cross-sectional area of our SM muscle $\left(17.5 \mathrm{~mm}^{2}\right)$ produced quite a limited density of force $13.7 \mathrm{mN} / \mathrm{mm}^{2}$ which should not produce excessive or damaging tension on muscle fibers.

Normalized force by cross-section area in our rat SM muscle was $54 \mathrm{mN} / \mathrm{mm}^{2}\left(940 \mathrm{mN} / 17.5 \mathrm{~mm}^{2}\right)$, which is lower than that obtained from the rabbit sternocleidomastoid muscle as reported by Falkenberg et al. [23]. In the rabbit maximal tetanic force of sternocleidomastoid muscle (during stimulation with $1 \mathrm{~s}$ train of $0.3 \mathrm{~ms}$ pulses at $100 \mathrm{~Hz}$ ) was about $4.5 \mathrm{~N}$ whereas a cross-section area of the muscle 
was $39 \mathrm{~mm}^{2}$, which results in a muscle force density of $115 \mathrm{mN} / \mathrm{mm}^{2}$.

4.2. Stimulation Intensity-Force Relationship. Our results showed that SM muscle force in the rat grows with stimulation current until about $0.1-0.2 \mathrm{~mA}$ when it reaches a plateau. The threshold of stimulation current, which can generate muscle contraction with noticeable force, is about $0.03 \mathrm{~mA}$. Muscle force characteristics were repeatable across different animals and therefore can be used as a normal control reference in reinnervation studies.

In many previous muscle force studies, due to the simplicity of the stimulator's circuitry and the necessity for safety during nerve stimulation (limited maximal amplitude of stimulation), the nerves were stimulated with rectangular pulses with regulated voltage. For example, Yoshimura et al. $[21,24]$ stimulated the peroneal nerve with bipolar silver electrodes and recorded muscle force from the extensor digitorum longus in the rat. The authors used a $250 \mathrm{~ms}$ train of $0.2 \mathrm{~ms}$ pulses with a regulated voltage between 2 and $6 \mathrm{~V}$. Cheng et al. [40] used a $30 \mathrm{~V}$ train of $0.2 \mathrm{~ms}$ pulses at $100 \mathrm{~Hz}$ to stimulate the femoral nerve and record force from the rectus femoris muscle in rabbits. The amplitude of stimulation pulses, which produced maximal active muscle force, was influenced by electrode placement on the nerve and varied radically across the different muscles and species used in those studies.

Stimulation with regulated current provides more reproducible results than stimulation with regulated voltage. Regardless of electrode impedance, a reproducible electric field can be created within stimulated tissue [41]. Therefore, we used stimulation with regulated current in the present study. Roszek et al. [29] stimulated the ischiadic nerve with bipolar silver electrodes and recorded force from the medial gastrocnemius muscle in a rat. They used $200 \mathrm{~ms}$ trains of $0.1 \mathrm{~ms}$ pulses of $3 \mathrm{~mA}$ current at different frequencies. Gradation in stimulation frequency from 15 to $100 \mathrm{~Hz}$ produced gradation in muscle force. Frieswijk et al. [42] searched for the threshold current for a single $0.1 \mathrm{~ms}$ pulse (monopolar stimulation of peroneal nerve with $\mathrm{NiCr}$ wire), which can generate a minimal muscle twitch response in the extensor digitorum longus in the rat. They found that the threshold current can be as low as $0.0026 \mathrm{~mA}$.

On the basis of the discussed results, we selected the optimal circumstances (initial passive tension and electrical stimulation parameters) for the rat SM muscle to contract with maximal force. This maximal muscle force will be used as a target level in our further study of muscle force recovery in a denervated SM muscle where different reinnervation techniques will be compared.

4.3. Stimulation Frequency-Force Relationship. Our experiments demonstrated that SM muscle force grows with the frequency of pulses until about $100-200 \mathrm{~Hz}$ when it reaches a plateau. A further increase of stimulation frequency produces a slight decrease of muscle force. The muscle force fusion frequency is higher than $50 \mathrm{~Hz}$. The relatively high frequency of tetanic fusion might result from muscle fiber type composition. The SM muscle is a fast muscle with over $80 \%$ of type II fibers (in rats Luff [43] in rabbits and primates McLoon [44] as well as our unpublished data in rats). Our results from the rat SM muscle are in agreement with those from other muscles or species used in previous studies. For instance, Devrome and MacIntosh [45] analyzed the force-frequency relationship for a rat gastrocenemius muscle with sciatic nerve stimulation using a $100 \mathrm{~ms}$ train of $0.05 \mathrm{~ms}$ pulses at a frequency up to $200 \mathrm{~Hz}$. They found the shape of the muscle force curve, which is similar to that observed in the present study with the highest muscle force at $200 \mathrm{~Hz}$. Interestingly, for this frequency of stimulation $(200 \mathrm{~Hz})$ muscle force was also significantly less sensitive to repetitive fatiguing contractions than for a lower frequency of stimulation $(<100 \mathrm{~Hz})$. This finding would indicate that the stimulation train of $200 \mathrm{~Hz}$ might be preferable to use (generating stronger and less variable contractions than stimulation at $100 \mathrm{~Hz}$ ) in our further study evaluating the level of tetanic force recovery in a reinnervated muscle. Roszek et al. [29] showed an interaction between the frequency of gastrocnemius stimulation and the length-force characteristics (shifting optimal muscle length to longer values with low frequency of stimulation $(<50 \mathrm{~Hz}))$. However, with a higher frequency of stimulation this shift of optimal length was minimal. Therefore, $200 \mathrm{~Hz}$ would be preferable over lower frequencies of stimulation to eliminate this confounding variable.

The results of Roszek et al. point out that the commonly used procedure of establishing optimal muscle length only once at the beginning of the study with a particular frequency of stimulation might not be appropriate when the muscle force is later analyzed at different frequencies. Repetitive testing to reveal the force-length relationship at all studied frequencies seems to be more appropriate. However, the analysis of Roszek et al. also showed that the optimal muscle length changes significantly with the frequency of stimulation only when the stimulation frequency is very low-below $40 \mathrm{~Hz}$. There was no significant difference in optimal muscle length between stimulation at $50 \mathrm{~Hz}$ and $100 \mathrm{~Hz}$. Stimulation frequencies below $40 \mathrm{~Hz}$ were not the focus of the present study, because one of our main goals was to establish optimal stimulation parameters which would generate a frequency fused contraction of maximal force in the SM muscle. Our investigations showed that muscle contraction to a train of stimulation pulses starts to be fused at about $50 \mathrm{~Hz}$ (see Figure 5). At this frequency muscle force reaches only about $60 \%$ of maximal force reached at $100-200 \mathrm{~Hz}$ (see Figures 6 and 7). Our study was focused at higher frequencies of stimulation where the change in optimal muscle length for maximal muscle force was not significant.

Brooks et al. [46] compared the force-frequency relationships between slow soleus and fast extensor digitorum longus (EDL) muscles in mice. Isometric force grew faster and reached a plateau at about $110 \mathrm{~Hz}$ for the soleus muscle (with a possible slight decline at $250 \mathrm{~Hz}$ ), but was still growing with the frequency set at $350 \mathrm{~Hz}$ in the case of the EDL muscle. These observations once again indicate that maximal muscle force is produced with a high frequency 
of stimulation and that the optimal frequency reflects the proportion of fast to slow fibers. Marsh et al. [47] studied the force-frequency relationship in the rat tibialis anterior with stimulation of the peroneal nerve with a $250 \mathrm{~ms}$ train of pulses of different frequencies $(50-300 \mathrm{~Hz})$. The authors found a similar relationship between force and frequency as observed in the present study (with decreased force at higher frequencies). The stimulation frequency, which generated maximum muscle force, decreased slightly in older animals. The strongest muscle contraction was produced at $200 \mathrm{~Hz}$ in young rats (3 months old) and at $150 \mathrm{~Hz}$ in older rats (1.52.5 years old). Older (as well as injured) rats also showed "tetanic fade" (rapid decrease of muscle contraction despite continued stimulation) at a higher frequency of stimulation. The tetanic fade was particularly pronounced at the highest frequency $(300 \mathrm{~Hz})$ used by the authors. Therefore, in our further study on muscle reinnervation, the $200 \mathrm{~Hz}$ would be a preferable stimulation frequency over $300 \mathrm{~Hz}$ to achieve maximal muscle force and to reduce the influence of tetanic fade as confounding factor.

Falkenberg et al. [23] examined twitch and tetanic force generation in the sternocleidomastoid muscle in rabbits (by stimulation of the spinoaccessory nerve). The muscle was stimulated with a $1 \mathrm{~s}$ train of $0.3 \mathrm{~ms}$ pulses with a growing frequency until $100 \mathrm{~Hz}$. Muscle force was the largest for $100 \mathrm{~Hz}$ but the force might be still larger if the authors would have used a higher stimulation frequency. The single twitch muscle force was $0.44 \mathrm{~N} \pm 0.06$ and tetanic muscle force was about $10 \times$ larger for stimulation with a $100 \mathrm{~Hz}$ train of pulses. The present study showed a similar relationship between tetanic and twitch force in the rat. Interestingly, Falkenberg et al. [23] also noticed that muscle force induced by direct muscle stimulation is similar to that induced by nerve stimulation. These findings are consistent with our results obtained from the rat $\mathrm{SM}$ muscle.

To produce tetanic contraction, we used a $200 \mathrm{~ms}$ train of biphasic pulses of $0.2 \mathrm{~ms}$ width, typically at $200 \mathrm{~Hz}$. Different frequencies of stimulation were used only when the influence of frequency of stimulation on muscle force was directly studied. This $200 \mathrm{~ms}$ time ensured that muscle force reached a plateau (see Figures 3 and 5). A similar duration (250$300 \mathrm{~ms}$ ) of the train of pulses (with pulse width $0.5 \mathrm{~ms}$ at $100 \mathrm{~Hz}$ ) was used by Cairns and Dulhunty [48] for the fast twitch SM muscle. The authors used a much longer-1s train of pulses to ensure a force plateau in the slow twitch soleus fibers.

Shin et al. [25] looked for the optimal stimulation parameters to produce maximal muscle force in the rat tibialis anterior. The authors showed a large standard deviation (35-50\%) of these parameters, not only between rats but also within each rat. Our data confirm this large variability of optimal stimulation parameters in the SM muscle.

Integrated EMG activity is sometimes used as a nondirect method of muscle force evaluation during muscle contraction. However, gross movement of the EMG electrode caused by SM muscle contractions during obstructive breathing (as well as during electrical stimulation) might be the source of unpredictable electrical artifacts causing a lack of good correlation between integrated EMG and muscle contractions [2]. Therefore, direct measurement of muscle force with a force transducer is an irreplaceable method to accurately measure muscle function.

\section{Conclusions}

Optimization of the variables affecting the isometric tetanic force of the rat SM muscle resulted in a choice of the following stimulation parameters to produce maximal tetanic force: $0.08 \mathrm{~N}$ (moderate) passive tension before stimulation, $0.2 \mathrm{~mA}$ stimulation current (with biphasic $0.2 \mathrm{~ms}$ width pulse), and $150 \mathrm{~Hz}$ stimulation frequency.

\section{Grants}

This research was supported by NIH Grant 5R01DC008599 from the National Institute on Deafness and Other Communication Disorders (to Liancai Mu).

\section{Acknowledgment}

The authors are grateful to Drs. Hungxi Su and Xiaolin Zhang for their excellent technical assistance during the surgical operations and data collection.

\section{References}

[1] D. Costa, M. Vitti, D. T. Oliveira, and R. P. Costa, "Participation of the sternocleidomastoid muscle on deep inspiration in man. An electromyographic study," Electromyography and Clinical Neurophysiology, vol. 34, no. 5, pp. 315-320, 1994.

[2] A. J. Raper, W. T. Thompson Jr., W. Shapiro, and J. L. Patterson, "Scalene and sternomastoid muscle function," Journal of Applied Physiology, vol. 21, no. 2, pp. 497-502, 1966.

[3] A. Legrand, E. Schneider, P.-A. Gevenois, and A. De Troyer, "Respiratory effects of the scalene and sternomastoid muscles in humans," Journal of Applied Physiology, vol. 94, no. 4, pp. 1467-1472, 2003.

[4] A. L. Hudson, S. C. Gandevia, and J. E. Butler, "The effect of lung volume on the co-ordinated recruitment of scalene and sternomastoid muscles in humans," Journal of Physiology, vol. 584, no. 1, pp. 261-270, 2007.

[5] A. De Troyer, P. A. Kirkwood, and T. A. Wilson, "Respiratory action of the intercostal muscles," Physiological Reviews, vol. 85, no. 2, pp. 717-756, 2005.

[6] J. Hamilton, S. Avitia, and R. F. Osborne, "Designing a bipedicled sternocleidomastoid muscle flap for parotidectomy contour deformities," Ear, Nose and Throat Journal, vol. 85, no. 1, pp. 20-21, 2006.

[7] V. Kumar, U. Gaud, M. Shukla, and M. Pandey, "Sternocleidomastoid island flap preserving the branch from superior thyroid artery for the reconstruction following resection of oral cancer," European Journal of Surgical Oncology, vol. 35, no. 9, pp. 1011-1015, 2009.

[8] L. Kaser and M. Muntener, "Delayed muscle fiber transformation after foreign-reinnervation of excessive muscle tissue," The Anatomical Record, vol. 223, no. 3, pp. 347-355, 1989.

[9] H. M. Tucker, "Reinnervation of the unilaterally paralyzed larynx," Annals of Otology, Rhinology and Laryngology, vol. 86, no. 6, pp. 789-794, 1977. 
[10] D. Meikle, R. E. Trachy, and C. W. Cummings, "Reinnervation of skeletal muscle: a comparison of nerve implantation with neuromuscular pedicle transfer in an animal model," Annals of Otology, Rhinology and Laryngology, vol. 96, no. 2, pp. 152157, 1987.

[11] S. K. Lee and S. W. Wolfe, "Peripheral nerve injury and repair," The Journal of the American Academy of Orthopaedic Surgeons, vol. 8, no. 4, pp. 243-252, 2000.

[12] B. J. F. Wong and R. L. Crumley, "Nerve wound healing: an overview," Otolaryngologic Clinics of North America, vol. 28, no. 5, pp. 881-895, 1995.

[13] H. M. Tucker, "Human laryngeal reinnervation," Laryngoscope, vol. 86, no. 6, pp. 769-779, 1976.

[14] H. M. Tucker, "Reinnervation of the paralyzed larynx: a review," Head and Neck Surgery, vol. 1, no. 3, pp. 235-242, 1979.

[15] R. L. Crumley, "Experiments in laryngeal reinnervation," Laryngoscope, vol. 92, pp. 1-27, 1982.

[16] G. S. Goding, C. W. Cummings, and D. A. Bright, "Extension of neuromuscular pedicles and direct nerve implants in the rabbit," Archives of Otolaryngology-Head and Neck Surgery, vol. 115, no. 2, pp. 217-223, 1989.

[17] N. D. Hogikyan, M. M. Johns, P. R. Kileny, M. Urbanchek, W. R. Carroll, and W. M. Kuzon Jr., "Motion-specific laryngeal reinnervation using muscle-nerve-muscle neurotization," Annals of Otology, Rhinology and Laryngology, vol. 110, no. 9, pp. 801-810, 2001.

[18] M. J. Bolesta, W. E. Garrett, B. M. Ribbeck, R. R. Glisson, A. V. Seaber, and J. L. Goldner, "Immediate and delayed neurorrhaphy in a rabbit model: a functional, histologic, and biochemical comparison," Journal of Hand Surgery, vol. 13, no. 3, pp. 352-357, 1988.

[19] L. K. Kalliainen, P. S. Cederna, and W. M. Kuzon Jr., "Mechanical function of muscle reinnervated by end-to-side neurorrhaphy," Plastic and Reconstructive Surgery, vol. 103, no. 7, pp. 1919-1927, 1999.

[20] P. S. Cederna, M. K. H. Youssef, H. Asato, M. G. Urbanchek, and W. M. Kuzon Jr., "Skeletal muscle reinnervation by reduced axonal numbers results in whole muscle force deficits," Plastic and Reconstructive Surgery, vol. 105, no. 6, pp. 2003-2009, 2000.

[21] K. Yoshimura, H. Asato, S. S. Jejurikar, P. S. Cederna, M. G. Urbanchek, and W. M. Kuzon Jr., "The effect of two episodes of denervation and reinnervation on skeletal muscle contractile function," Plastic and Reconstructive Surgery, vol. 109, no. 1, pp. 212-219, 2002.

[22] M. G. Urbanchek, D. E. Ganz, M. A. Aydin, J. H. van der Meulen, and W. M. Kuzon Jr., "Muscle-nerve-muscle neurotization for the reinnervation of denervated somatic muscle," Neurological Research, vol. 26, no. 4, pp. 388-394, 2004.

[23] J. H. Falkenberg, P. A. Iaizzo, and L. K. McLoon, "Muscle strength following direct injection of doxorubicin into rabbit sternocleidomastoid muscle in situ," Muscle and Nerve, vol. 25, no. 5, pp. 735-741, 2002.

[24] K. Yoshimura, H. Asato, P. S. Cederna, M. G. Urbanchek, and W. M. Kuzon Jr., "The effect of reinnervation on force production and power output in skeletal muscle," Journal of Surgical Research, vol. 81, no. 2, pp. 201-208, 1999.

[25] R. H. Shin, T. Vathana, G. A. Giessler, P. F. Friedrich, A. T. Bishop, and A. Y. Shin, "Isometric tetanic force measurement method of the tibialis anterior in the rat," Microsurgery, vol. 28 , no. 6 , pp. 452-457, 2008.
[26] D. Cantillon and A. Bradford, "Effects of age and gender on rat upper airway muscle contractile properties," Journals of Gerontology Series A, vol. 55, no. 8, pp. B396-B400, 2000.

[27] J. Lannergren and H. Westerblad, "The temperature dependence of isometric contractions of single, intact fibres dissected from a mouse foot muscle," Journal of Physiology, vol. 390, pp. 285-293, 1987.

[28] Y. Ishii, T. Watari, and T. Tsuchiya, "Enhancement of twitch force by stretch in a nerve-skeletal muscle preparation of the frog Rana porosa brevipoda and the effects of temperature on it," Journal of Experimental Biology, vol. 207, no. 26, pp. 45054513, 2004.

[29] B. Roszek, G. C. Baan, and P. A. Huijing, "Decreasing stimulation frequency-dependent length-force characteristics of rat muscle," Journal of Applied Physiology, vol. 77, no. 5, pp. 2115-2124, 1994.

[30] D. E. Rassier, B. R. MacIntosh, and W. Herzog, "Length dependence of active force production in skeletal muscle," Journal of Applied Physiology, vol. 86, no. 5, pp. 1445-1457, 1999.

[31] P. M. Rack and D. R. Westbury, "The effects of length and stimulus rate on tension in the isometric cat soleus muscle," Journal of Physiology, vol. 204, no. 2, pp. 443-460, 1969.

[32] J. Celichowski, K. Grottel, and E. Bichler, "Differences in the profile of unfused tetani of fast motor units with respect to their resistance to fatigue in the rat medial gastrocnemius muscle," Journal of Muscle Research and Cell Motility, vol. 20, no. 7, pp. 681-685, 1999.

[33] M. M. Johns, M. Urbanchek, D. B. Chepeha, W. M. Kuzon Jr., and N. D. Hogikyan, "Length-tension relationship of the feline thyroarytenoid muscle," Journal of Voice, vol. 18, no. 3, pp. 285-291, 2004.

[34] J. Krier, R. A. Meyer, and W. H. Percy, "Length-tension relationship of striated muscle of cat external anal sphincter," American Journal of Physiology, vol. 256, no. 4, pp. 773-778, 1989.

[35] K. Floyd and J. F. B. Morrison, "The mechanical properties of oesophageal striated muscle in the cat and sheep," Journal of Physiology, vol. 248, no. 3, pp. 717-724, 1975.

[36] M. J. Kim, W. S. Druz, and J. Danon, "Mechanics of the canine diaphragm," Journal of Applied Physiology, vol. 41, no. 3, pp. 369-382, 1976.

[37] K. K. McCully and J. A. Faulkner, "Length-tension relationship of mamalian diaphragm muscles," Journal of Applied Physiology, vol. 54, pp. 1681-1686, 1983.

[38] G. A. Farkas and D. F. Rochester, "Contractile characteristics and operating lengths of canine neck inspiratory muscles," Journal of Applied Physiology, vol. 61, no. 1, pp. 220-226, 1986.

[39] J. Davis, K. R. Kaufman, and R. L. Lieber, "Correlation between active and passive isometric force and intramuscular pressure in the isolated rabbit tibialis anterior muscle," Journal of Biomechanics, vol. 36, no. 4, pp. 505-512, 2003.

[40] N. Cheng, L. Xuexiang, and A. Huang, "Experimental comparison of muscle contractility after three methods of reinnervation," Annals of Plastic Surgery, vol. 33, no. 2, pp. 166-170, 1994.

[41] J. P. Reilly, Applied Bioelectricity: From Electrical Stimulations to Electropathology, Springer, New York, NY, USA, 1998.

[42] T. A. Frieswijk, J. P. A. Smit, W. L. C. Rutten, and H. B. K. Boom, "Force-current relationships in intraneuronal stimulation: role of extraneural medium and motor fibre clustering," Medical and Biological Engineering and Computing, vol. 36, pp. 422-430, 1998. 
[43] A. R. Luff, "Dynamic properties of fiber bundles from the rat sternomastoid muscle," Experimental Neurology, vol. 89, no. 3, pp. 491-502, 1985.

[44] L. K. McLoon, "Muscle fiber type compartmentalization and expression of an immature myosin isoform in the sternocleidomastoid muscle of rabbits and primates," Journal of the Neurological Sciences, vol. 156, no. 1, pp. 3-11, 1998.

[45] A. N. Devrome and B. R. MacIntosh, "The biphasic forcevelocity relationship in whole rat skeletal muscle in situ," Journal of Applied Physiology, vol. 102, no. 6, pp. 2294-2300, 2007.

[46] S. V. Brooks, J. A. Faulkner, and D. A. McCubbrey, "Power outputs of slow and fast skeletal muscles of mice," Journal of Applied Physiology, vol. 68, no. 3, pp. 1282-1285, 1990.

[47] D. R. Marsh, L. R. Hinds, W. S. Lester, B. E. Reinking, and F. W. Booth, "The force-frequency relationship is altered in regenerating and senescent rat skeletal muscle," Muscle and Nerve, vol. 21, no. 10, pp. 1265-1274, 1998.

[48] S. P. Cairns and A. F. Dulhunty, "The effects of betaadrenoreceptor activation on contraction in isolated fast- and slow-twitch skeletal muscle fibers of the rat," British Journal of Pharmacology, vol. 110, pp. 1133-1141, 1993. 

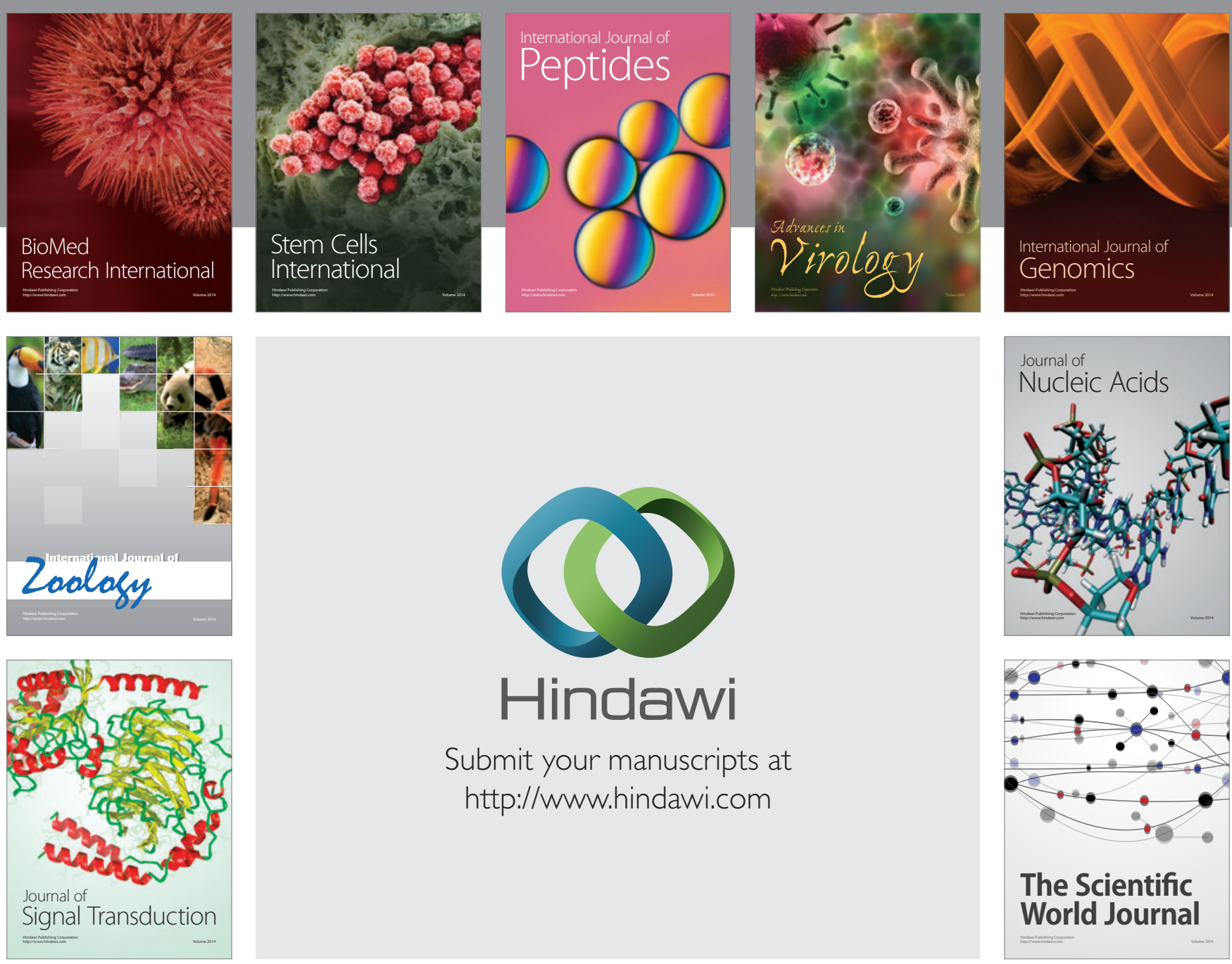

Submit your manuscripts at

http://www.hindawi.com
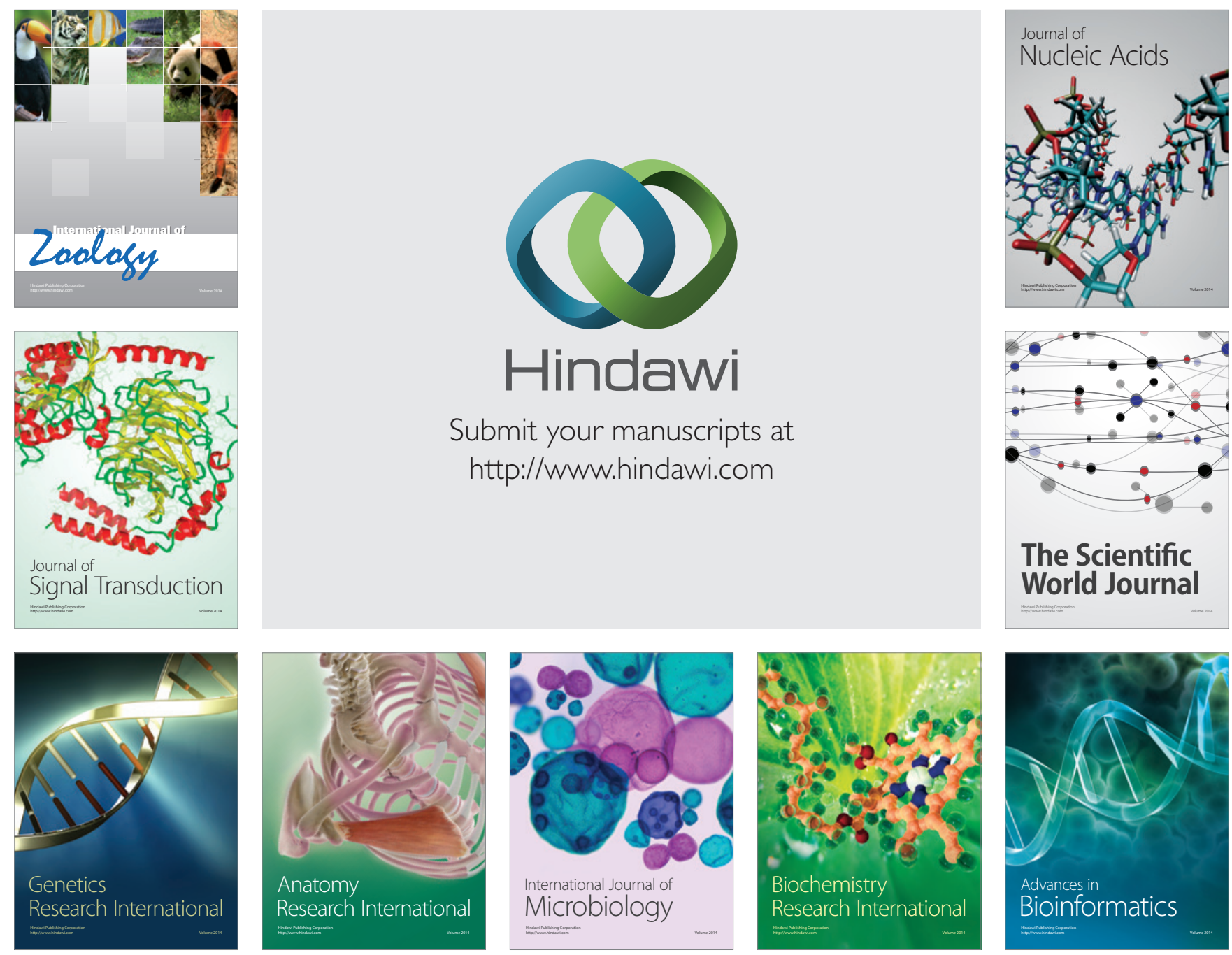

The Scientific World Journal
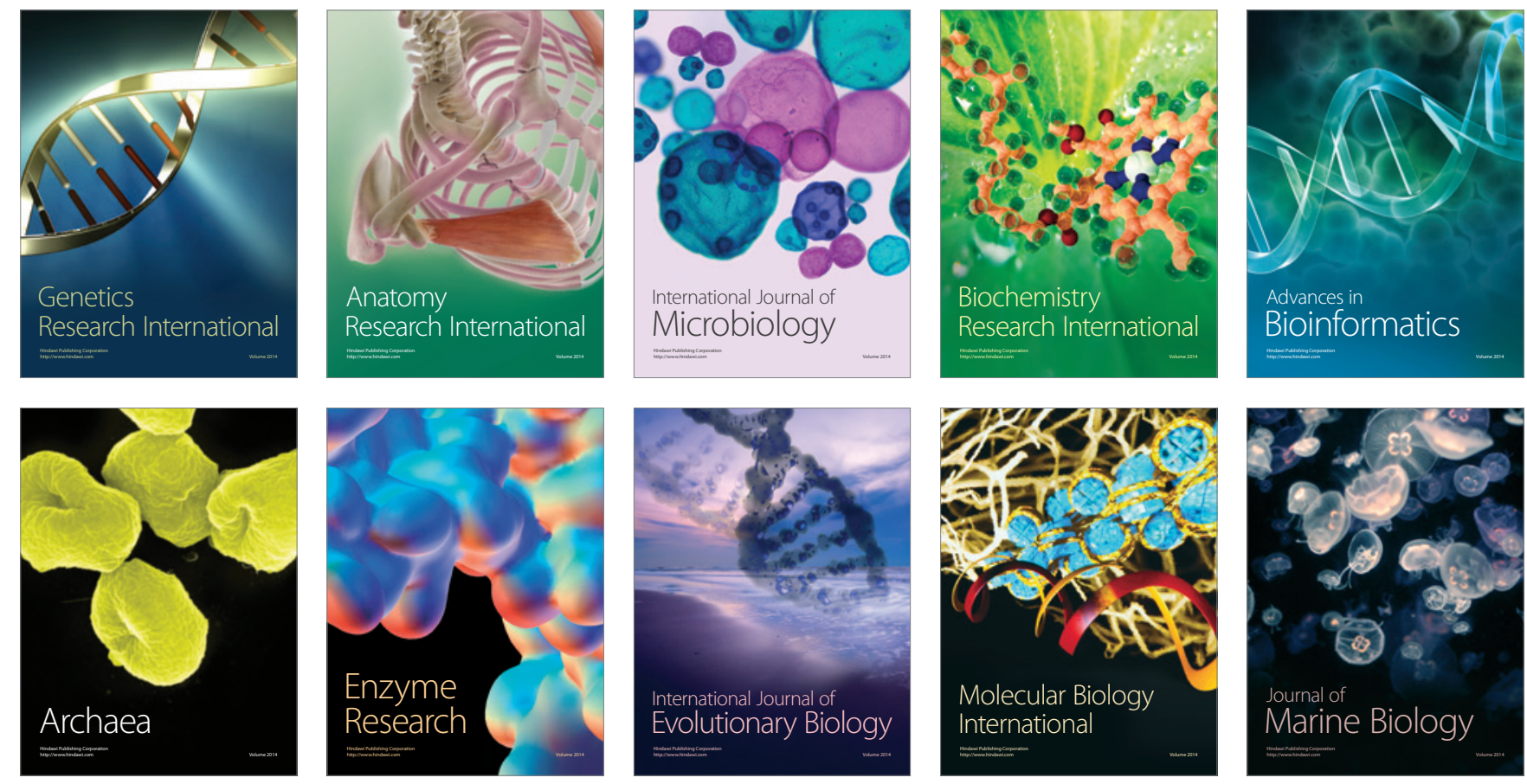